\title{
Mechanical and Electrical Properties of CdTe Tetrapods Studied by Atomic Force Microscopy
}

\author{
Liang Fang ${ }^{1}$, Jeong Young Park ${ }^{1}$, Yi Cui ${ }^{2}$, Paul Alivisatos ${ }^{2}$, \\ Joshua Shcrier $^{3}$, Byounghak Lee ${ }^{3}$, Lin-Wang Wang ${ }^{3}$, and Miquel Salmeron ${ }^{1 *}$ \\ ${ }^{1}$ Molecular Foundry, Materials Science Division, Lawrence Berkeley National \\ Laboratory, One Cyclotron Road, Berkeley, California 94720 \\ ${ }^{2}$ Chemistry Department, University of California at Berkeley, Berkeley, California 94720 \\ ${ }^{3}$ Computational Research Division, Lawrence Berkeley National Laboratory, One \\ Cyclotron Road, Berkeley, California 94720
}

*Author to whom correspondence should be addressed. Electronic mail: mbsalmeron@lbl.gov

\begin{abstract}
The mechanical and electrical properties of CdTe tetrapod-shaped nanocrystals have been studied with atomic force microscopy. Tapping mode images of tetrapods deposited on silicon wafers revealed that they contact the surface with the ends of three arms. The length of these arms was found to be $130 \pm 10 \mathrm{~nm}$. A large fraction of the tetrapods had a shortened vertical arm as a result of fracture during sample preparation. Fracture also occurs when the applied load is a few nanonewtons. Compression experiments with the AFM tip indicate that tetrapods with the shortened vertical arm deform elastically when the applied force was less than $50 \mathrm{nN}$. Above 90 $\mathrm{nN}$ additional fracture events occurred that further shorted the vertical arm. Loads above $130 \mathrm{nN}$ produced irreversible damage to the other arms as well. Current-voltage characteristics of tetrapods deposited on gold indicated semiconducting behavior with a current gap of $\sim 2 \mathrm{eV}$ at low loads $(<50 \mathrm{nN})$ and a narrowing to about $1 \mathrm{eV}$ at loads between 60 and $110 \mathrm{nN}$. Atomic calculation of the deformation suggests that the ends of the tetrapod arms are stuck during compression so that the deformations are due to bending modes. The reduction of the current gap is due to electrostatic effects, rather than strain deformation effects inside the tetrapod.
\end{abstract}




\section{Introduction}

CdSe and CdTe nanocrystals possess interesting photo-electronic properties (1) that make them interesting materials for solar cell applications (2). Branched tetrapods with a CdSe core and terminal CdTe branches are also important due to unusual charge-separation properties (3-5). In addition to electronic properties, the peculiar shape of the nanocrystals might confer to these materials interesting mechanical properties, such as large compliance and toughness, with potential applications as shock absorbers.

In this paper we present a study of the mechanical properties of individual CdTe tetrapods, including adhesion, compliance and resistance to fracture. We will also present some results on the relationship between mechanical and electronic properties.

\section{Methods}

\section{A. Experimental}

CdTe tetrapods were synthesized using colloid-chemistry (2). The crystals were suspended in toluene with a concentration of $1 \mu \mathrm{M}$. The solution was used without further purification. For measurements of mechanical properties the samples were spin-casted from the toluene suspension onto a silicon wafer at a speed of 8000 RPM, followed by washing with pure methanol. For electrical measurements the tetrapods were deposited on a (111) oriented gold film on a glass substrate following a similar spin casting procedure. The films were cleaned with solvents and annealed with a butane flame prior to deposition.

To study the mechanical properties of the tetrapods the force-volume (FV) technique was used (6-8). In this technique force-distance curves are acquired at each pixel of a selected area imaged by the AFM. From these curves adhesion and elasticity maps can be produced. The measurements were carried out with an Asylum Research MFP-3D AFM. The piezo scanner was calibrated in $\mathrm{x}, \mathrm{y}$, and $\mathrm{z}$ directions using NIST certified calibration gratings (MikroMasch). The spring constants of rectangular Silicon cantilevers (Olympus) were determined from the thermal noise spectrum and found to range from 1.2 to $1.7 \mathrm{~N} / \mathrm{m}$. All experiments were performed under ambient conditions $\left(21 \pm 1^{\circ} \mathrm{C}, 30 \%\right.$ relative humidity). A loading speed of $200 \mathrm{~nm} / \mathrm{s}$ was used in all force-distance measurements.

The electrical measurements were carried out in an RHK AFM operating in ultra-high vacuum using cantilevers with a conductive TiN coating (9). The spring constant of these cantilevers was measured to be $2.5 \mathrm{~N} / \mathrm{m}$ using the method of Sader et al. (10).

\section{B. Theoretical}


To simulate the mechanical properties of the tetrapods, we used the valence force field (VFF) method containing nearest-neighbor bond-stretching, bond-angle bending and bondlength/bond-angle terms fitted to the experimental bulk elastic constants.(11) This type of classical model cannot handle bond breaking, therefore our calculations only concern the elastic regime in the experiment. The elastic constants $\left(\mathrm{C}_{11}, \mathrm{C}_{12}, \mathrm{C}_{44}\right)$ of our VFF model: $(5.48,3.58$, 1.89) $x 10^{10} \mathrm{~N} \mathrm{~m}^{-2}$ agree well with the experimental values $(5.35,3.71,2.02) \times 10^{10} \mathrm{~N} \mathrm{~m}^{-2}$ of bulk CdTe (12). To model the compression of the tetrapod the top and bottom boundaries of the system were treated as impenetrable planes perpendicular to the vertical arm of the tetrapod, which were then pushed together, minimizing the VFF total energy with respect to all atomic coordinates. We examined two possible cases: (i) the horizontal arms are allowed to slide freely on the bottom plane, modeling a scenario in which there is no sticking interaction with the surface; and (ii) the ends of the horizontal arms are fixed in place, modeling a scenario in which strong interaction with the surface prevents the ends of the arms from sliding. The numerical derivative of the energy (with respect to vertical displacement), gives the force.

We consider a CdTe tetrapod with arm diameter of $2.8 \mathrm{~nm}$, horizontal arm lengths of $38 \mathrm{~nm}$, and vertical arm length of $10.5 \mathrm{~nm}$ (containing 24,692 cation and anion atoms), i.e., the dimensions were reduced by a factor of three from the experimental one while keeping the same shape and aspect ratios. This is a compromise between a making the system size large enough to be physically relevant and the computational cost, especially for the subsequent quantum mechanical calculations. Since the displacement distances will be smaller, we will instead compare the spring constants (force/displacement); and because the dimensional scaling is expected to follow the Young's equation scaling, we will include a multiplicative factor of 3 when comparing to the experimental results.

To calculate the electronic structure of the tetrapod we fitted a CdTe empirical pseudopotential with a band gap of $1.54 \mathrm{eV}$, effective mass of $0.125 \mathrm{~m}$, and spin-orbit splitting of $0.87 \mathrm{eV}$. The conduction band and valence band deformation potentials of the pseudopotential are fitted to abinitio results (13). The band edge states of the tetrapod under various compressive loads are calculated using the folded spectrum method (14) based on this empirical pseudopotential Hamiltonian.

\section{Results}




\section{A. Geometrical structure of the tetrapods on Si wafers}

Fig. 1a shows $2.4 \mu \mathrm{m} \times 2.4 \mu \mathrm{m}$ tapping mode image of isolated tetrapods on a silicon wafer. The tetrapods are standing upright with three arms contacting the surface and the fourth arm pointing vertically out. Instabilities in the feedback control were observed during tapping to

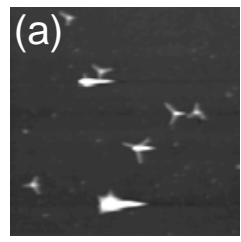

(c)

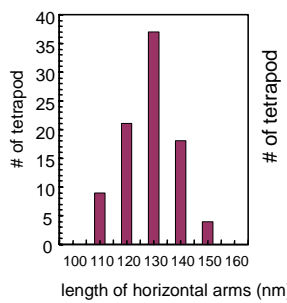

(e)

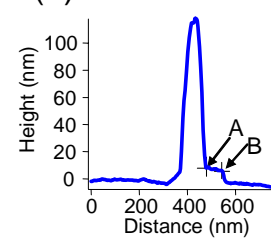

(f)

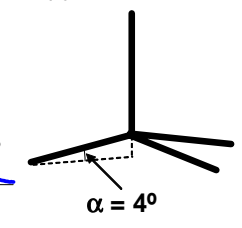

Figure 1. L. Fang et al. the tip of the arm, which give rise to the bright spot in the images near the center of the tetrapod. This instability might be related with the easy bending deformation of the long arm. The length of the three base arms was found to be $130 \pm 10 \mathrm{~nm}$ (see histogram in fig. 1c), while the vertical arm was about $120 \mathrm{~nm}$. However, tetrapods with four long and nearly equal arms like those in figure $1 \mathrm{a}$ and $\mathrm{b}$ were found to be uncommon. More often the images revealed tetrapods with a substantially shortened vertical arm. The most common height is $30 \pm 10 \mathrm{~nm}$, followed by $70 \mathrm{~nm}$, as shown in the histogram in fig. 1d. Since the core of the tetrapod is 10 to $15 \mathrm{~nm}$ above the surface, the actual arm length is also shorter by that amount. An image of the more common, short arm tetrapod is shown in fig. 2a. We hypothesize that fracture of the vertical arm occurs as a result of capillary forces acting while drying.

Line profiles over tetrapods both long and short (figs. 1e and 2b), show that the base arms are closer to the substrate than expected from a perfect tetragonal geometry. The slope of the arm between point $\mathrm{A}$ and $\mathrm{B}$ is about 0.06 , corresponding to an angle with the substrate of $\sim 4^{\circ}$ (fig. $1 \mathrm{f}$ ). This value should be compared with $19.5^{\circ}$ expected for undistorted tetrahedrons. In this geometry the gap between the center of the tetrapod and the substrate is about 10 nanometers. The distortion is probably the result of strong attractive forces with the substrate that bend the arms and increase the contact length.

\section{B. Mechanical properties}

The mechanical response of the tetrapods to applied loads was investigated using forcevolume (FV) mapping with a pixel density chosen so that at least one force-distance curve is taken over the top of the vertical arm in each tetrapod. An adhesion or pull-off force map from these curves is shown in fig. 2c. The adhesion of the tip is lower on the tetrapods (by about 10 
$\mathrm{nN}$, see histogram in d), than on the silicon substrate. This is not surprising because: a) the tetrapods are covered by a layer of trioctylphosphine oxide and octadecylphosphonic acid, which
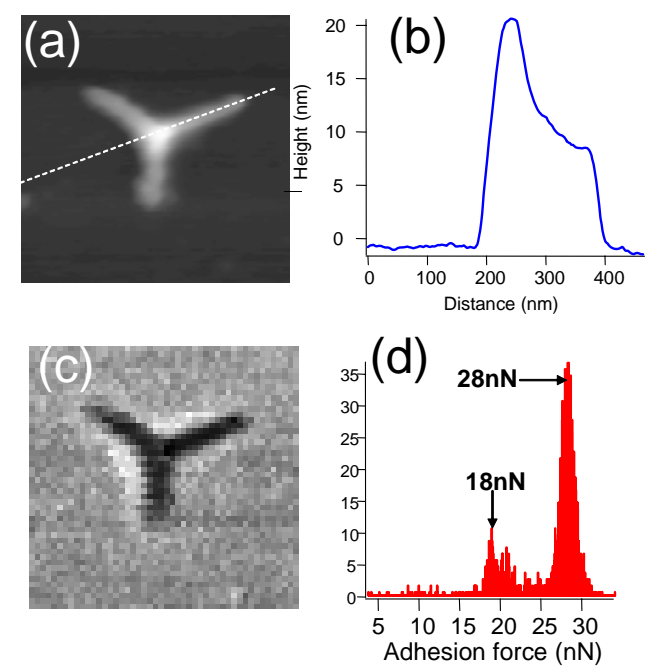

Figure 2. L. Fang et al. terminate with hydrophobic and weakly interacting $\mathrm{CH}_{3}$ groups while the $\mathrm{Si}$ substrate is hydrophillic, so that in air an additional capillary adhesion force is present; b) the contact area between the AFM tip and the tetrapods is small, because the tip radius is about $40-60 \mathrm{~nm}$ and the arms have a diameter of 8 $\mathrm{nm}$.

To determine the elastic or inelastic response of the tetrapods experiments were performed at increasing values of the maximum load. Fig. 3a shows a $2.4 \mu \mathrm{m} \times 2.4 \mu \mathrm{m}$ tapping mode image of an area containing several tetrapods, with vertical arm lengths in the range of 30-60 nm After an FV experiment with maximum load of $50 \mathrm{nN}$ the same area was re-imaged and is shown in Fig. 3b. The tetrapods appear broader now due to an increase in the tip radius. It was often found that sharp tips ( $<10 \mathrm{~nm}$ radius) giving the highest resolution images break easily upon application of forces above a few tens of nanonewtons. The heights are now close to $25 \mathrm{~nm}$, indicating that loads of $50 \mathrm{nN}$ are sufficient to break the vertical arm. Since the contact area between tip and tetrapod is essentially determined by the diameter of the vertical arm $(8 \mathrm{~nm})$, the ratio $50 \mathrm{nN} /$ $\pi .(4 \mathrm{~nm})^{2}=\sim 1 \mathrm{GPa}$ puts a higher bound to the elastic deformation limit of shortened vertical arms. A second FV experiment was performed with maximum load of $90 \mathrm{nN}$. Fig. 3c shows an image obtained after this experiment. Except for some drift, the image is similar to the previous one and the height of the tetrapod is unchanged. However, as the applied force continues to increase the rest of tetrapod structure finally collapses. This occurred after the third FV experiment at a load of $130 \mathrm{nN}$. Fig. 3d shows the image obtained after such experiment.

More quantitative observations can be made by examination of individual force-distance curves, as shown in figure 4 . In these curves the
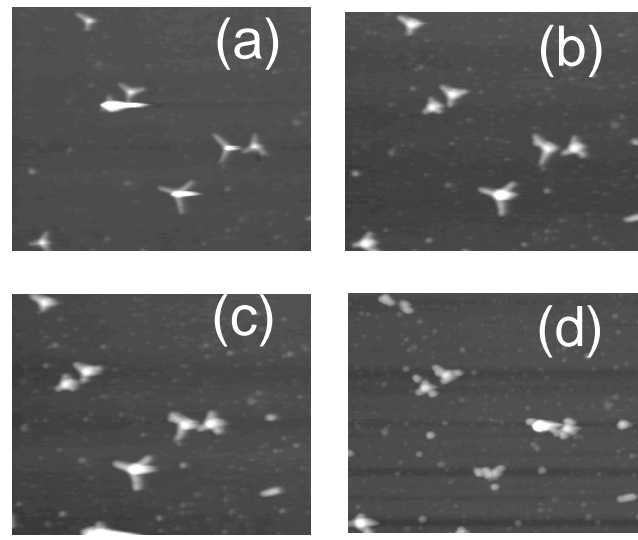

Figure 3. L. Fang et al. distance origin was arbitrarily placed at the 
minimum of the approach curve. The initial height of the tetrapod in the image of Fig. 4a (enclosed in a circle) was $21 \mathrm{~nm}$. Three force-separation curves on top of the tetrapod with maximum load values as in the previous experiment (50, 90 and $130 \mathrm{nN}$ ) were obtained and are shown in fig. 4b, c and d. Separation (x-axis) in these curves is defined as the difference between the advance of the sample and the bending of the lever, thus corresponding to actual compression of the sample. At a load of $50 \mathrm{nN}$ (fig. $4 \mathrm{~b})$, the tetrapod has been compressed by $4 \mathrm{~nm}$. The retract curve in the same graph shows some hysteresis as well as a higher adhesion (pull-off) force. After release of the load the height recovered to $21 \mathrm{~nm}$. The difference between the height under compression and the height under zero load (4 $\mathrm{nm})$ corresponds to the elastic deformation, which can be attributed to flattening of the pyramid formed by the three base arms. The spring constant for this tetrapod deformation mode can thus be
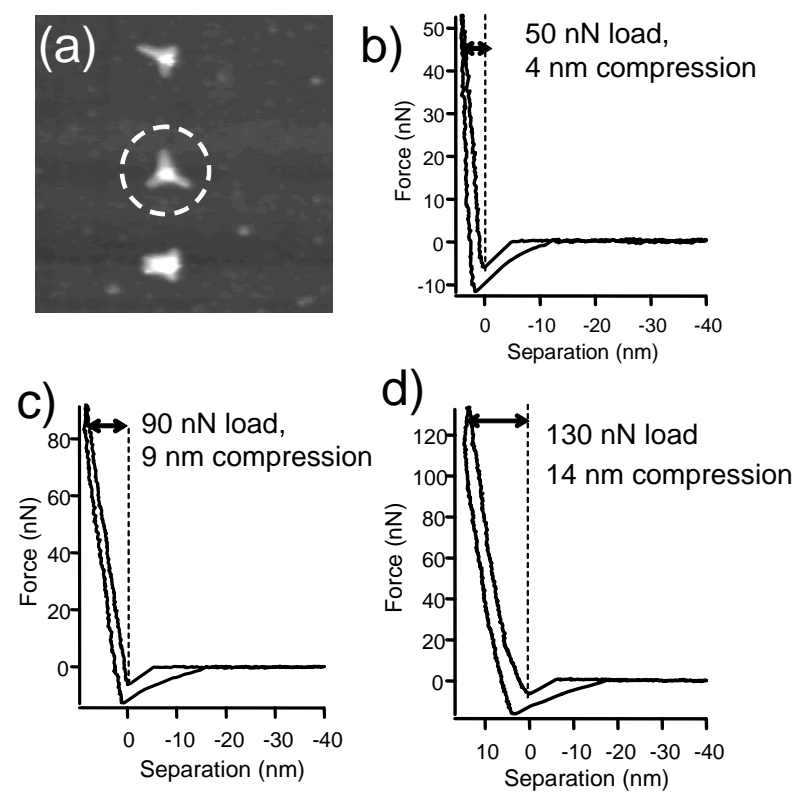

Figure 4. L. Fang et al. estimated to be $50 \mathrm{nN} / 4 \mathrm{~nm}=12.5$ $\mathrm{N} / \mathrm{m}$.

When the maximum load was $90 \mathrm{nN}$ in the second FV experiment, the force-separation curve on same tetrapod shows a compression of $9 \mathrm{~nm}$. After release of the load the height recovered again to its initial value of $21 \mathrm{~nm}$. We attribute the $9 \mathrm{~nm}$ elastic deformation again to flattening of the base of the pyramid. The same arithmetic as before gives a spring constant of 90 $\mathrm{nN} / 9 \mathrm{~nm}=10 \mathrm{~N} / \mathrm{m}$, not too far from the previous estimate.

The third F-V experiment to a maximum load of $130 \mathrm{nN}$ produced a compression of $15 \mathrm{~nm}$. As shown in fig. 3 these high load values produced irreversible damage to the base arms of the tetrapod. A higher limit for the plastic yield stress for this deformation can thus be set to $130 \mathrm{nN}$ $/ \pi .(4 \mathrm{~nm})^{2}=2.6 \mathrm{GPa}$.

Our first simulation considered the case of a tetrapod in which the horizontal base arms are allowed to freely move along the surface, shown as the dashed line in Fig.5. The force increases linearly as the base arms are completely flattened against the surface, with a calculated spring constant of $0.07 \mathrm{~N} / \mathrm{m}$. In the inset figures, we depict representative structures from these 
simulations, color coding the local hydrostatic strain at each atom site. The greatest compressive and expansive changes occur near the core joining the arms together, and the horizontal base arms are relatively uncompressed except near the tip. Scaling the spring constant by 3 (as discussed in Sect II.B) gives a result of $0.21 \mathrm{~N} / \mathrm{m}$, which is $\sim 5$ times smaller than the experimental value. Since the AFM imaging performed in Sect. III A might not resolve an enlarged zinc-blende core of the tetrapod, we have examined how this might change the forces. Increasing the diameter of the zinc-blende core by as much as a factor of 1.5 (while keeping the diameter of the arms fixed) did not alter the force behavior significantly, so we eliminate this possibility.

Next we examined the case in which the horizontal base arm ends were fixed horizontally in place, as might result from the strong sticking interaction with the

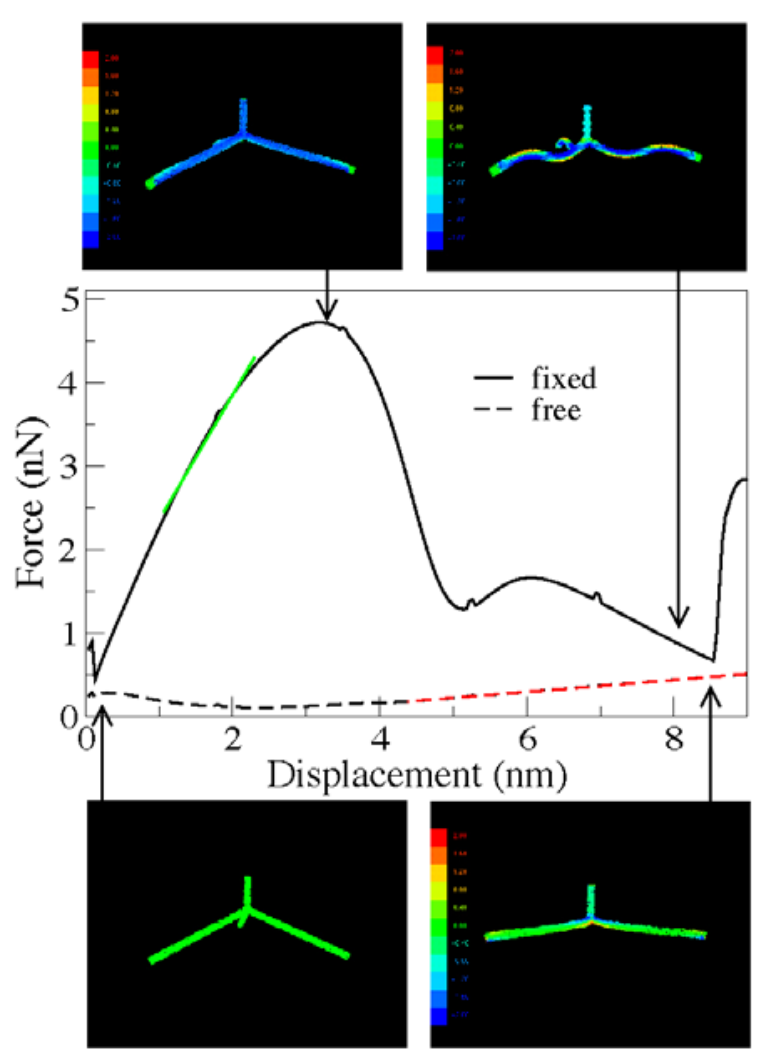

Figure 5. L. Fang, et.al substrate mentioned in Sect III. A. The results of this simulation are shown as the solid line in Fig.5. Unable to slide along the surface, the horizontal arms undergo an S-shaped buckling. For the displacements up to $\sim 3.3 \mathrm{~nm}$, this would correspond to a spring constant of $1.5 \mathrm{~N} / \mathrm{m}$, leading to a scaled spring constant for the tetrapod of $\sim 4.5 \mathrm{~N} / \mathrm{m}$, which is more consistent with the experimental results than the freely sliding case. Beyond $3.3 \mathrm{~nm}$ displacement, the force is observed to be reduced, but this probably corresponds to the plastic regime observed in the experiment, and would be accompanied by other structural effects which are beyond the scope of our elastic simulation. The fact that our calculated spring constant is about a factor of 2 smaller than the experimental one might have several causes. One possible reason is that in our calculation, we have ignored any capillary force between the tetrapod and the substrate. As a result, our starting point (with zero load) is a perfect tetragon, not a distorted one as is found in the experiment.

\section{Electrical conductance of tetrapods under load}


Fig. 6a shows a topographic image of a region in a Au film containing a tetrapod. Its vertical arm is $23 \mathrm{~nm}$ high. Using a conductive TiN-coated tip, the current through the tetrapod was measured as a function of applied bias, with the tip contacting the top of the tetrapod's vertical arm at a load below $0 \mathrm{nN}$ (i.e., pulling the tip away from the surface but still in the attractive well). The current bias characteristic under this condition is shown in fig. 5b, both in I$\mathrm{V}$ and $\mathrm{dI} / \mathrm{dV}$ forms (the later in a log scale). These I-V curves were quite reproducible indicating that the contact is elastic at these loads. The current become immeasurably small $(<0.1 \mathrm{pA})$ when the applied voltage between tip and gold decreased below \pm 1.1 volt. This $2.2 \mathrm{eV}$ range of current gap is higher than the optical bandgap of CdTe $(1.6-1.8 \mathrm{eV})$ reported by L. Manna et al. (15). In general the measured current gap is not necessarily equivalent to the semiconductor energy gap because in a diode configuration the field distribution in the gap between tip and gold electrodes is unknown. In addition, Schottky barriers and poorly defined interfaces of the electrodes with the surfactant covered tetrapods make the $2.2 \mathrm{eV}$ current gap an upper limit of semiconductor energy gap.

This gap was found nonetheless to depend on applied load as shown in figure 6c. For small elastic deformations, i.e. for effective loads below $50 \mathrm{nN}$ (pressure $<1 \mathrm{GPa}$ ), the gap remains approximately constant. Between $50 \mathrm{nN}$ and $70 \mathrm{nN}$, when the pyramidal structure is elastically flattened, the gap decreases to about $0.9 \mathrm{eV}$. Finally, at high load (> $100 \mathrm{nN}$ ), the I-V measurement shows a metallic character suggesting that the tetrapod has been damaged and that the tip contacts the gold substrate. This is consistent with the observation in fig. 3d where the horizontal arms were damaged by a load of $130 \mathrm{nN}$.

We have calculated the conduction band minimum (CBM) and valence band maximum (VBM) states using the empirical pseudopotential Hamiltonian. We have calculated three loading situations: one with zero load (bottom left inset in Fig.5, to be called
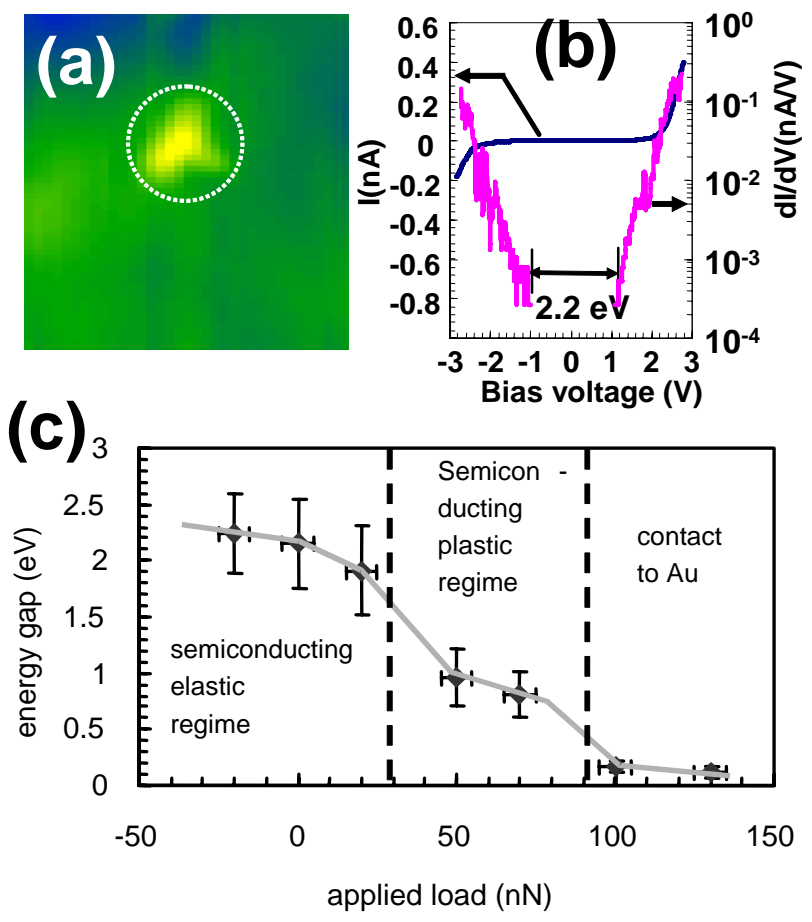

Figure 6. L. Fang et al. 
free tetrapod below); one at the maximum load with fixed arm ends (top left inset in Fig.5, to be called fixed arm tetrapod below); and one with the arm touching the substrate in the free sliding case (bottom right inset in Fig.5, to be called free sliding tetrapod below). The VBM energies for these three cases are: $-3.997,-3.965,-4.017 \mathrm{eV}$ respectively, while the CBM energies for these three cases are: $-2.002,-1.911,-1.957 \mathrm{eV}$ respectively. Thus the band gap changes from $1.995 \mathrm{eV}$ in the free tetrapod, to $2.054 \mathrm{eV}$ in the fixed arm tetrapod, to $2.06 \mathrm{eV}$ in the free sliding tetrapod. The VBM and CBM wavefunctions for the free tetrapod and the free sliding tetrapod are plotted in Fig.7, while the case for the fixed arm tetrapod is very similar to that of the free tetrapod, thus

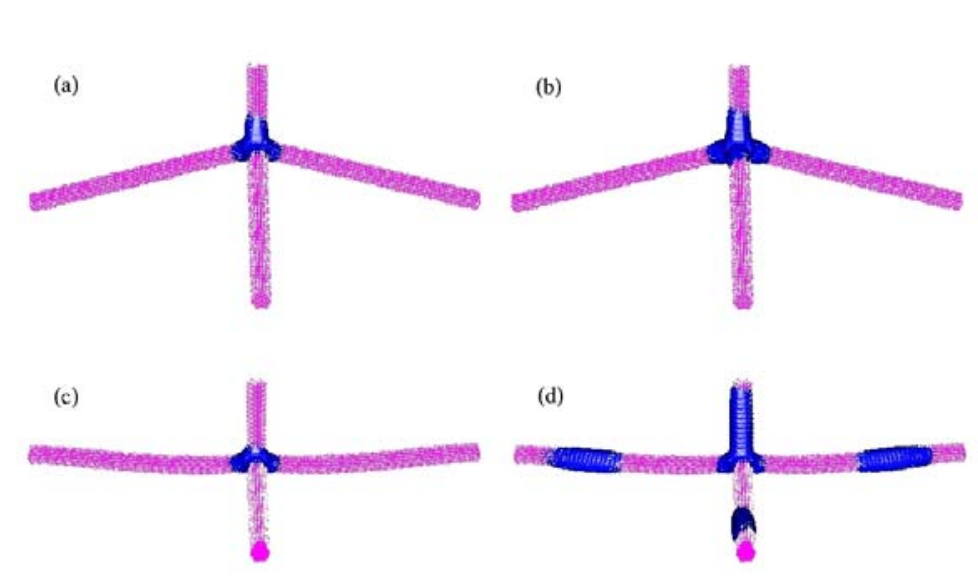

Figure 7, L. Fang, et.al is not shown here. We see that both VBM and CBM wavefunctions are localized near the core of the tetrapod, except for the CBM of the free sliding tetrapod, where $70 \%$ of the weight of the charge density is located at the arms. What happen is that the $\mathrm{CBM}+1$ state in the free tetrapod is located at

the three arms (16) and it has the same symmetry as the CBM at the core. In the free sliding tetrapod, the core region has a strong compressive hydrostatic strain, which raises the energy of the original CBM at the core. As a result, this CBM and the CBM+1 states hybridize, and form the current CBM with both core and the arm charge. It is at a anti-crossing transition stage of these two states. Thus our calculation indicates that it might be possible to use mechanical load to cause state crossing. Note that for larger tetrapods (as in the experiments reported here), this state crossing becomes easier since the original CBM to CBM+1 energy difference is smaller, but the strain effects remain the same.

The band gap change from $1.995 \mathrm{eV}$ to $2.06 \mathrm{eV}$ is due to the internal strain of the tetrapod, caused by the application of load. It does not explain the large current band gap changes observed in Fig.6(C), and it has the wrong sign (band gap increases, instead of decreases). One possible reason for the current band gap decreasing is due to the polarization potential $\mathrm{P}(\mathrm{r})$ in the quasi-particle equation. $\mathrm{P}(\mathrm{r})$ is defined as $0.5 \sum_{r^{\prime} \rightarrow r}\left[W\left(r^{\prime}, r\right)-W_{b}\left(r^{\prime}, r\right)\right]$, where $\mathrm{W}\left(\mathrm{r}^{\prime}, \mathrm{r}\right)$ is the 
screened potential at $r^{\prime}$ of a point charge at $r$ in the given system, and $\mathrm{W}_{b}\left(r^{\prime}, r\right)$ is the same screened potential in an infinite bulk. It has been shown that (17) $\mathrm{P}(\mathrm{r}$ ) needs to be added (subtracted) to the pseudopotential Hamiltonian used above to get the quasi-particle equation for the electron (hole). In the above calculation, $\mathrm{P}(\mathrm{r})$ is not included. However, due to the existence of the metallic substrate, there could be a large negative $\mathrm{P}(\mathrm{r})$. Since the wavefunctions are localized near the core, the magnitude of $\mathrm{P}(\mathrm{r})$ at the core can provide an estimate for the eigenenergy change. For a metallic substrate $\mathrm{P}(\mathrm{r})$ is caused by the image charge. Thus, if the height of the core is $h, P(r)=-1 / 4 h$. Then the change on the quasiparticle band gap is $2 P(r)=-1 / 2 h$. A typical tetrapod will have $\mathrm{h}=25 \mathrm{~nm}$ when $\sim 20 \mathrm{nN}$ negative load is applied, this will yield a band gap reduction of $\sim$ $30 \mathrm{meV}$. When large load $(\sim 30 \mathrm{nN})$ is applied (near the limit of the elastic regime in Fig.6(c)), the core of the tetrapod is probably close to touch the substrate. Then $\mathrm{h}=4 \mathrm{~nm}$ for an $8 \mathrm{~nm}$ diameter tetrapod. This will give a band gap reduction of $\sim 0.2 \mathrm{eV}$. This can be used to explain the $\sim 0.2 \mathrm{eV}$ band gap reduction in the elastic regime of Fig.6(c). After the tetrapod completely touches the substrate (perhaps, in the plastic regime of Fig.5(c)), the above perturbation argument might no longer hold, and more detailed calculations based on the P(r) added Schrodinger's equation become necessary. In those cases, the wavefunction might become further localized due to $\mathrm{P}(\mathrm{r})$, and make the band gap even smaller. Other plastic change of the tetrapod shapes and atomic structures can also cause large changes in the band gap.

\section{Conclusions}

We have used AFM to study the mechanical and electronic properties of individual tetrapodshaped CdTe nanocrystals. The tetrapods were found to be very fragile and many "as deposited" tetrapods have their vertical arm considerably shortened, fractured we believe by capillary forces during solvent evaporation. We also found that such fracture events result in a distribution of vertical heights with three most likely values of 30, 70 and $120( \pm 10) \mathrm{nm}$.

We have shown that the vertical arm can be also broken by the AFM tip when the applied force reaches a few tens of nano-newtons. An upper limit of $1 \mathrm{GPa}$ was found for the elasticinelastic threshold leading to tetrapod fracture. After the vertical arm is reduced to a few tens of $\mathrm{nm}$, the remaining pyramidal structure behaves as a spring with a constant of approximately 10 $\mathrm{N} / \mathrm{m}$. The deformation occurs by flattening of the pyramid while maintaining the base arms intact. The tetrapod structure (horizontal arms) collapses at pressures below 2.6 GPa. Our simulations indicate that whether or not the ends of the arms are allowed to slide freely on the substrate plays an important role in determining the spring properties in the elastic regime. By comparing the 
calculated and the observed spring constants, we concluded that the ends of the arms are probably stuck during the compression process.

Electrical transport measurements of tetrapods on a gold substrate indicate that they behave as semiconductors with an apparent energy gap of $2.2 \mathrm{eV}$ or less at pressures below $1 \mathrm{GPa}$ (corresponding to a $50 \mathrm{nN}$ load). This gap decreases to about $0.9 \mathrm{eV}$ in the pressure range of 1 to $2 \mathrm{GPa}$ (60-110 $\mathrm{nN}$ loads), when the pyramidal structure is deformed. At higher loads, the contact has a metallic character, due to the tip making contact with the gold substrate. Atomistic quantum mechanical calculations show that band gap changes due to the elastic deformation of the tetrapod are much too small to explain the observed band gap change in the elastic regime. Instead, an electrostatic polarization potential due to the image charge of the metallic substrate might be used to explain the observed large band gap reduction. Besides, our calculations also indicate that mechanical loads might be used to induce electron state crossing in the tetrapod.

\section{Acknowledgements}

This work was supported by the Director, Office of Energy Research, Office of Basic Energy Sciences, Molecular Foundry, Materials Sciences Division, of the U.S. Department of Energy through the Lawrence Berkeley National Laboratory, Contract No. DE-AC0205CH11231. The calculations were performed using the resources of the National Energy Research Scientific Computing Center. We thank Drs. Peter Graf and Qingzhong Zhao (National Renewable Energy Laboratory) for providing the code to generate the uncompressed tetrapod geometries. 


\section{References:}

[1] J.S. Liu, T. Tanaka, K. Sivula, A.P. Alivisatos, J.M.J. Frechet, Journal of the American Chemical Society 126 (2004) 6550-6551.

[2] D.J. Milliron, S.M. Hughes, Y. Cui, L. Manna, J.B. Li, L.W. Wang, A.P. Alivisatos, Nature 430 (2004) 190-195.

[3] L.W. Wang, Journal of Physical Chemistry B 109 (2005) 23330-23335.

[4] J.B. Li, L.W. Wang, Applied Physics Letters 85 (2004) 2929-2931.

[5] H. Yu, J.B. Li, R.A. Loomis, L.W. Wang, W.E. Buhro, Nature Materials 2 (2003) 517-520.

[6] E. A-Hassan, W.F. Heinz, M.D. Antonik, N.P. D'Costa, S. Nageswaran, C.A. Schoenenberger, J.H. Hoh, Biophysical Journal 74 (1998) 1564-1578.

[7] M. Radmacher, M. Fritz, J.P. Cleveland, D.A. Walters, P.K. Hansma, Langmuir 10 (1994) 3809-3814.

[8] C. Rotsch, M. Radmacher, Langmuir 13 (1997) 2825-2832.

[9] J.Y. Park, R.J. Phaneuf, D.F. Ogletree, M. Salmeron, Appl. Phys. Lett. 86 (2005) 172105.

[10] J.E. Sader, J.W.M. Chon, P. Mulvaney, Rev. Sci. Instrum 70 (1999) 3967.

[11] A. J. Williamson, L. W. Wang, A. Zunger, Physical Review B 62 (2000) 1296312977.

[12] U. Rossler (ed), Semiconductors, II-VI and I-VII Compounds: Semimagnetic Compounds, vol 41B of Landolt-Bornstein New Series, Group III (Springer, Heidelberg, 1999)

[13] S.-H. Wei, A. Zunger, Phys. Rev. B 60, 5404 (1999).

[14] L. W. Wang, A. Zunger, J. Chem. Phys. 100, 2394 (1992).

[15] L. Manna, D.J. Milliron, A. Meisel, E.C. Scher, A.P. Alivisatos, Nature Materials 2 (2003) 382-385.

[16] J. Li, L. W. Wang, Nano Lett. 3, 1357 (2003).

[17] L.W. Wang, J. Phys. Chem. B 109, 23330 (2005). 


\section{Figure Captions}

Figure 1. (a) Tapping mode AFM image $(2.4 \mu \mathrm{m} \times 2.4 \mu \mathrm{m})$ showing several tetrapods on a silicon wafer. The bright spot near the tetrapod center is due to feedback response to mechanical instabilities. (b) Tapping mode image $(600 \mathrm{~nm}$ x $600 \mathrm{~nm}$ ) of a single tetrapod and cursor profile along the broken line (e). (c), (d) histogram of arm lengths found in the images. The histogram shows that tetrapods with long vertical arms $(\sim 120 \mathrm{~nm})$ are minority, the majority having heights in the range from 20 to $50 \mathrm{~nm}$ and a few with a length of $70 \mathrm{~nm}$. (f) Model geometry derived from the height profile. The slope of the near horizontal arms (from A to B) is small, about $4^{\circ}$, indicating that the center of the tetrapod is separated from the substrate by $7-9 \mathrm{~nm}$.

Figure 2 (a) Topographical image of a tetrapod with a height of $25 \mathrm{~nm}$, and height profile (b). (c) Adhesion force map extracted from the pull-off forces in a 64x64 pixel Force-Volume experiment. (d) Histogram of adhesion forces showing a bimodal distribution.

Figure 3 Tapping mode AFM images $(2.4 \mu \mathrm{m} \times 2.4 \mu \mathrm{m})$ taken before force-volume (FV) measurements. (a) Initial image before application of forces. The tetrapod heights vary between 30 and $60 \mathrm{~nm}$. (b) After a FV scan with maximum load of $50 \mathrm{nN}$. (c) After a FV scan at $90 \mathrm{nN}$. (d) Same after $130 \mathrm{nN}$. At this load the base arm structure is damaged.

Figure 4. (a) Tapping mode image $(1.5 \mu \mathrm{m} \times 1.5 \mu \mathrm{m})$ of three tetrapods with heights of 24,25 , and $25 \mathrm{~nm}$. (b-d) Force-separation curves taken over the center of the tetrapod marked by the white circle in (a). The maximum load is 50,90 and $130 \mathrm{nN}$ in each curve respectively, which produced compressions of 4, 9 and 14 nm respectively.

Figure 5. Calculated force-displacement relationship. The figures represent the tetrapods with color code to indicate local hydrostatic strain at each atom site. Green means zero hydrostatic strain, red means tensile strain, and blue means compressive strain. The dashed line in the graph at the center corresponds to the free sliding arm case; the solid line corresponds to the fixed arm end case. The tetrapod using in the calculations is 3 times smaller in each dimension compared to a typical experimental tetrapod.

Figure 6. (a) Contact mode AFM topographical image $(1.0 \mu \mathrm{m} \times 1.0 \mu \mathrm{m})$ obtained in ultra high vacuum, of a tetrapod deposited on a $\mathrm{Au}(111)$ oriented film. The height is $25 \mathrm{~nm}$. (b) Currentvoltage (I-V) and dI/dV plots at an effective applied load of $0 \mathrm{nN}$. (c) Plot of current gap width as a function of effective applied load. In the left region the tetrapod deforms elastically by flattening of the pyramidal base. In the center region the load causes the tetrapod arms to be flattened against the substrate. The right hand region corresponds to damage of the tetrapod and tip-gold metallic contact.

Figure 7. The wavefunction iso-density plots for: (a) free, uncompressed tetrapod; (b) pinned tetrapod as shown in the bottom left inset in Fig.5; (c) free sliding, compressed tetrapod; and (d) flattened free sliding tetrapod as in the bottom right inset in Fig.5. For (a) and (c) the wavefunction corresponds to VBM states. For (b) and (d) to CBM states. The same isosurface value is used in all plots. 